\title{
Análisis de integración de la cadena de suministros en la industria textil en Ecuador. Un caso de estudio
}

\author{
Analysis of the integration of the supply chain in the textile \\ industry in Ecuador. A case study
}

\author{
Neyfe Sablón-Cossío ${ }^{1 *} \quad$ Erik Orozco Crespo ${ }^{2} \quad$ Alexander Pulido-Rojano $^{3}$ \\ Ana Julia Acevedo-Urquiaga ${ }^{4,5}$ Sebastiana del Monserrate Ruiz Cedeño ${ }^{6}$
}

Recibido 11 de diciembre de 2018, aceptado 12 de enero de 2020

Received: December 11, 2018 Accepted: January 12: 2020

\begin{abstract}
RESUMEN
En la actualidad, la integración en la cadena de suministro facilita el logro de la competitividad de sus actores. Es un factor importante cuando se trata de alcanzar objetivos comunes y altos grado de interacción dentro de cada eslabón de la cadena. En este estudio, se desarrolló un diagnóstico de la cadena de suministro de camisas en la zona norte del Ecuador y se plantearon un conjunto de estrategias y objetivos conjuntos para la mejora de la competitividad de los actores involucrados. Para ello, se diseñó un instrumento de verificación, conformado por las dimensiones de Estrategia, Información, Planeación, Compras, Inventario colaborativos, Transporte y Desempeño colaborativo, para evaluar la integración de 96 actores. La validez de constructo del instrumento fue realizada y la información fue procesada mediante la estadística descriptiva e inferencial. Los resultados de esta investigación reflejan el bajo nivel de integración de la cadena de suministro en estudio. Donde las variables de mayor debilidad se enfocan en: estrategia, información, compras e inventario colaborativos. Los aportes de la presente investigación se evidencian en su valor práctico, ya que los resultados obtenidos son utilizados para el diagnóstico, análisis y propuesta de estrategias y/o acciones para mejorar el desempeño de la industria textil en Ecuador.
\end{abstract}

Palabras clave: Cadena de suministro, estrategias de integración, logística colaborativa, estudio de integración.

\begin{abstract}
At present, the integration in the supply chain facilitates the achievement of the competitiveness of its actors. It is an important factor when trying to reach common goals and high degree of interaction within each link of the chain. In this study, a diagnosis of the shirt supply chain in the northern area of Ecuador was developed and a set of strategies and joint objectives were proposed to improve the competitiveness of the actors involved. For this purpose, a verification instrument was designed, consisting of the dimensions of Strategy, Information, Planning, Purchases, Collaborative Inventory, Transport and Collaborative Performance, to evaluate the integration of 96 actors. The construct validity of the instrument was carried out and the information was processed using descriptive and inferential statistics. Results of this research
\end{abstract}

1 Instituto de Posgrado. Grupo de Producción y Servicios. Universidad Técnica de Manabí. Portoviejo, Ecuador. E-mail: nsablon@utm.edu.ec; nsabloncossio@gmail.com

2 Carrera de Ingeniería Industrial. Universidad Técnica del Norte. Ibarra, Ecuador. E-mail: eorozco@utn.edu.ec

3 Departamento de Ingeniería Industrial. Universidad Simón Bolívar. Barrranquilla, Colombia.

E-mail: apulido3@unisimonbolivar.edu.co.

4 Laboratorio de Logística y Producción. Universidad Tecnológica de la Habana. Cuba. E-mail: anajuliaa@gmail.com

5 Programa de Ingeniería Industrial. Fundación Universitaria San Mateo. Bogotá, Colombia.

6 Carrera de Administración de Empresas. Universidad Técnica de Manabí. Portoviejo, Ecuador. E-mail: sruiz@utm.edu.ec

* Autor de correspondencia: nsablon@utm.edu.ec 
reflect the low level of integration of the supply chain under study. Where the weakest variables focus on: strategy, information, purchases and collaborative inventory. The contributions of this research are evidenced in its practical value, since the results obtained are used for the diagnosis, analysis and proposal of strategies or actions to improve the performance of the textile industry in Ecuador.

Keywords: Supply chain, integration strategies, collaborative logistics, integration study.

\section{INTRODUCCIÓN}

A nivel global, se manifiestan cambios en la forma de administrar y conformar los negocios. En la actualidad, el objetivo no es hacer un producto sino ser competitivo al elaborar alguna de sus partes. De esto se infiere la necesidad de interconexión entre varias empresas, entidades y organismos que se denominan actores de la cadena de suministro a la que pertenecen [1]. A ello se suma, las nuevas formas de producción: el desarrollo de nuevos materiales, en base a la elaboración y venta de proyectos personalizados a las necesidades de los clientes, así como la tecnología 3D. Esto enmarcado en las tecnologías de la información, las comunicaciones y el conocimiento infieren en la necesidad de integración de las cadenas de suministro a nivel internacional y nacional $[2,3]$.

En este sentido, concurren tres razones principales para la Integración de las Cadenas de Suministro: primero, es una necesidad en el contexto de los negocios, segundo se debe a que cada vez se encuentran menos integradas verticalmente, más especializados y tercero, se buscan proveedores que puedan garantizar el abastecimiento con componentes de alta calidad y a un bajo precio $[4,5]$.

Esta temática ha sido investigada desde diferentes enfoques, debido a la amplitud y complejidad del enfoque de integración en las cadenas de suministro. Por ejemplo, una metodología de gestión de inventario [6], la coordinación de interfuncional entre las compras y los suministros de actores [7], la integración de los elementos de calidad y los rendimientos en las cadenas de suministro [8]. Estos estudios, enfocados en una variable de integración en la cadena. Otros antecedentes, se enmarcan en la flexibilidad de la integración [9], como una característica particular de este concepto. Desde el análisis matemático, existen modelos de integración que se basan en la minimización del costo bajo circunstancias específicas [10]. Por otro lado, existe presencia de la integración en las prácticas verdes, desde la alineación de las actividades y los procesos [11] como del desarrollo de su gestión [12] para la sustentabilidad de la cadena.

En Ecuador, la integración de la cadena de suministros de la industria textil es una necesidad latente, sobre todo en el sector de distribución de camisetas de la zona geográfica del país. Esta necesidad se evidencia en aspectos tales como:

- Una baja participación en las exportaciones de los textileros ecuatorianos con aproximadamente el 1,3\% de las exportaciones totales del país y por la caída en las ventas en USD 81,5 millones en el 2016 en relación a las alcanzadas en el 2015, según datos del Instituto Nacional de Estadística y Censos (INEC).

- Altos inventarios de materia prima por trabajar de forma independiente en el mercado y la no inclusión de la relación oferta y demanda.

- Productos con poca variedad, y bajo valor agregado para el cliente.

- Deficiencias para entrar al mercado nacional e internacional por falta de interacción de los actores.

- Ausencia de relación entre actores (empresas) de la cadena de suministros con iguales o diferentes enfoques de negocios.

- Baja determinación de las necesidades de los clientes, por lo que existe un alto efecto látigo de la demanda.

- Poca eficiencia en los procesos internos de las empresas que influye en los altos precios de algunos productos.

- La planificación de la producción se concentra a nivel operativo y en menor medida a nivel táctico.

- Existe inercia a la hora de un cambio en las micro, pequeñas y medianas empresas (MIPYMES) del sector textil y en algunas empresas ante el mercado.

- La cercanía de países productores como Colombia, hace que tengan una alta competencia. 
Por todo lo anterior, el objetivo de la presente investigación es diagnosticar la integración de la cadena de camisetas del Ecuador mediante un instrumento de chequeo y su análisis descriptivo e inferencial para la definición de las estrategias y sus objetivos conjuntos. Este trabajo posee fundamentalmente, un valor práctico dado por la factibilidad de la aplicación de este estudio bajo las condiciones del sector textil ecuatoriano; y un valor metodológico que se manifiesta en la posibilidad de establecer una forma coherente un estudio de integración que permita tomar decisiones conjuntas en la cadena y como consecuencia, la mejora de las camisetas en función de las necesidades del cliente (valor social).

Este documento está estructurado de la siguiente manera: la sección 2 presenta un análisis teórico de la integración en la cadena de suministros, la sección 3 muestra los materiales y métodos para la evaluación de la integración de la cadena de suministros. Los resultados y el análisis se muestran en la sección 4. Finalmente, la sección 5 presenta las conclusiones de la presente investigación.

\section{LA INTEGRACIÓN EN LA CADENA DE SUMINISTRO}

Una de las bases de la integración en la cadena de suministro es su orientación hacia una filosofía que gestiona la coordinación del flujo total de un canal de distribución desde el proveedor hasta el cliente final [13]. Las definiciones del término Integración en la Cadena de Suministro (ICS) coinciden en dos aspectos: el primero, se relaciona con que se transite de la interrelación de los procesos y flujos internos de cada empresa, a la combinación de los procesos y flujos de múltiples actores; el segundo, que dichos procesos se alineen en función de la estrategia de la cadena, con el objetivo de satisfacer al cliente
[14]. Además, se conceptualizan las etapas de desarrollo de la integración en función del nivel de su complejidad [5, 15-19].

La Figura 1 presenta las etapas de integración en la cadena de suministro propuesta por $[5,18]$ : Asignación, Negociaciones de mercado, Asociación, Cooperación, Coordinación y Colaboración.

A continuación, se presenta una descripción general de cada etapa.

- Asignación: En donde se estipulan recursos desde una entidad estatal o institución.

- Negociaciones de mercado: Incluyen discusiones enfocadas hacia el precio y las relaciones como socios y adversarios.

- Asociación: Donde se establecen acuerdos económicos.

- Cooperación: Busca establecer contratos a largo plazo con pocos proveedores.

- Coordinación: Establece compartir información por las diferentes vías de tecnología de la información y las comunicaciones.

- Colaboración: Donde se manifiesta la integración de la cadena, se realiza la planificación conjunta y se comparte la tecnología [5]. Esta es la fase superior de la integración.

Además del nivel de integración en el que se ubique la cadena, se necesitan establecer los elementos básicos que satisfagan los comportamientos de intercambio en la organización y el uso de la tecnología [2]. Por otro lado, autores como [6] y [15] consideran que la calidad de las relaciones de los negocios que se establecen entre los socios, son esenciales para alcanzar la ICS y la instrumentación exitosa de mecanismos, estrategias y técnicas [6]; donde se requiere de un alto nivel de confianza, de compromiso y de mutua información entre los agentes de la cadena [15].

Fuente: Adaptado de [5, 18].

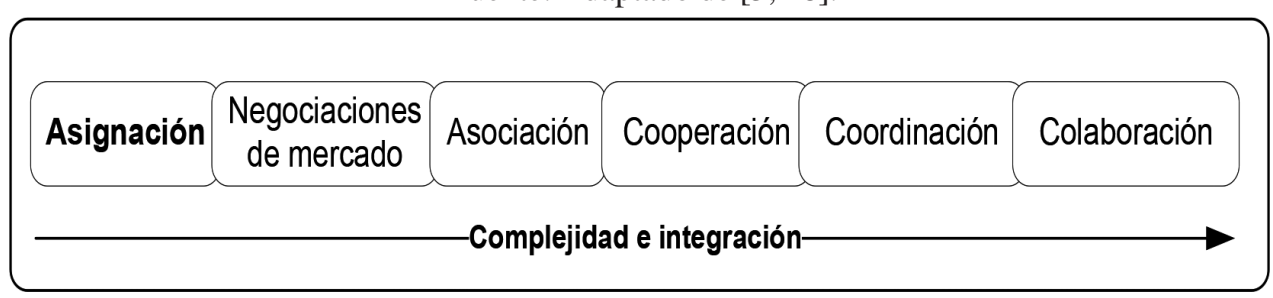

Figura 1. Etapas de integración en la cadena de suministro. 
La ICS tiene dos perspectivas: vertical y horizontal. En el primer caso, los nodos de la cadena aceptan el hecho de que sea una sola entidad (empresa virtual) recolecte y transmita la información a los demás nodos para que desarrollen los procesos correspondientes [20]. En segundo lugar, y como una tendencia más actual, se considera una colaboración de tipo horizontal, en donde cada nodo se relaciona con el que considere oportuno y, según las necesidades, crea relaciones de tipo colaborativa o no [16]. Un nodo de integración se define como un punto de interacción en las cadenas de suministro para obtener un resultado conjunto, donde intervienen varios actores que se interconectan por medio de los flujos materiales, informativos y financieros, para brindar productos y servicios a los clientes [14].

Además, la ICS puede clasificase como centralizada y descentralizada. En la primera, las decisiones se toman de forma independiente en cada uno de los nodos de ICS, mientras que en la segunda existe un gestor global que coordina las decisiones a lo largo de la cadena [21]. En ambas clasificaciones, las competencias laborales del potencial humano influyen en la toma de decisiones de cada actor y el éxito en la ICS.

La ICS se interrelaciona con las actividades estratégicas, tácticas y operativas para lograr la mayor rentabilidad posible a los integrantes de la cadena, desde el proveedor primario hasta el consumidor final $[1,15,17,19,22$ y 23], y de esto influye en la interdependencia entre los actores. Se asocia directamente a la interrelación en el proceso de planificación y control [24], con su mayor expresión en el modelo Scord 12,0 como modelo para la excelencia en la cadena. Este modelo, se despliega en los tres niveles de planeación mediante los procesos (el modelo se conforma por cuatro niveles), los indicadores y sus estándares, buenas prácticas y la tecnología, que permiten el control de la cadena [25]. Esto ha influido en que algunos autores, clasifiquen la integración según los niveles de planeación [19].

Otros se refieren a las dimensiones: integración de la información, coordinación y compartir recursos y las formas de relación entre las organizaciones [26]. Junto con la interrelación de la tecnología corriente arriba y abajo [2, 27-30]. En otra variante, se identifican las influencia entre los actores y eslabones de una cadena [31, 32], y se interrelacionan con el nivel de servicio al cliente [33]. Estos elementos necesitan de la cultura colaborativa, como base para el desarrollo de esta tendencia. Por ende, la formación del personal en estas habilidades y conocimientos forma parte de otras investigaciones.

En relación de este artículo con otras investigaciones, por ejemplo, el estudio realizado por [6], se diferencian en que este último se centra en el proceso de inventario colaborativo, definiendo políticas para la integración y colaboración, planificación colaborativa, integración de procesos claves y críticos, medición del desempeño y elaboración de planes de acción enmarcados en la gestión de inventarios [6]. Por su parte, este artículo presenta un análisis de integración de la cadena de suministros en la industria textil en Ecuador para la propuesta de estrategias de integración efectivas.

\section{MATERIALES Y MÉTODOS}

Con la meta de cumplir el objetivo de este artículo, se inició el estudio a partir de la aplicación del instrumento de chequeo [14], conformado por las dimensiones de planeación colaborativa, pronóstico colaborativo, reabastecer colaborativamente y desempeño colaborativo [34]. En aras de favorecer el carácter unidimensional del instrumento y de sus dimensiones, además, para mejorar la interpretación de los resultados, se decidió transformar el instrumento original llevándolo de 4 a 7 dimensiones, las cuales quedaron así: Estrategia $\left(\mathrm{D}_{1}\right)$, Información $\left(\mathrm{D}_{2}\right)$, Planeación $\left(\mathrm{D}_{3}\right)$, Compras $\left(\mathrm{D}_{4}\right)$, Inventario $\left(\mathrm{D}_{5}\right)$, Transporte $\left(\mathrm{D}_{6}\right)$ y Desempeño colaborativo $\left(\mathrm{D}_{7}\right)$.

Se empleó el modelo Alpha de Cronbach para medir la consistencia interna del instrumento [35], el cual revela el nivel de homogeneidad existente entre los elementos o items que componen cada dimensión. Este análisis sólo implicó a los elementos medidos de forma ordinal. Se analizó la opción descriptiva "si se elimina el elemento", centrando la atención en los incrementos de los valores de Alpha de Cronbach y en el coeficiente de determinación $\left(\mathrm{R}^{2}\right)$ para identificar a aquellos que menos se parecen al resto y que pudieran ser eliminados de la dimensión [36].

El Alpha de Cronbach se interpretó a partir de los intervalos: $\alpha>0,9$ excelente, $\alpha>0,8$ bueno, $\alpha>0,7$ 
aceptable, $\alpha>0,6$ cuestionable, $\alpha>0,5$ pobre y $\alpha<0,5$ inaceptable [37]. Además, de otras consideraciones en el marco de los estudios exploratorios que plantean que un valor de $\alpha$ cercano a 0,7 es adecuado y es el nivel mínimo aceptable [38]. En tal sentido, otros autores sugieren un $\alpha$ igual a 0,6 controlando que la dimensión no supere los 10 ítems.

Se adicionó el análisis descriptivo por dimensiones, desde $D_{1}$ hasta $D_{7}$. Los elementos nominales fueron descritos mediante sus frecuencias relativas, mientras que los ordinales fueron analizados a partir de sus medianas y de sus frecuencias relativas. También se obtuvieron elementos en forma de escala que resultaron de cálculos intermedios que posteriormente fueron categorizados y llevados nuevamente a una escala ordinal. El instrumento diseñado se muestra en la Tabla 1.

Donde:

- $\quad i\{i=1,2, \ldots k\}$ : Empresa $i$ analizada, donde $k$ representa el tamaño de la muestra seleccionada (número de empresas).

- $\quad \mathrm{D}_{\mathrm{n}}\{n=1,2, \ldots 7\}$ : Dimensión $n$ analizada del conjunto de dimensiones.

- $\quad \mathrm{E}_{\mathrm{n}, \mathrm{m}}\{m=1,2, \ldots l\}$ : ítems $m$ medidos dentro de la dimensión $n$, donde $l$ representa el número total de elementos medidos dentro de cada dimensión $n$.

- $\quad \mathrm{CD}_{\mathrm{n}}$ : Variable ordinal que evalúa la puntuación categorizada para la dimensión $n$.

- $\mathrm{CM}_{\mathrm{i}}$ : Conglomerado de pertenencia de la empresa $i$.

- $\mathrm{CS}_{\mathrm{i}}$ : puntuación total de colaboración obtenida para la empresa $i$.

- Mo: Moda calculada para cada $\mathrm{E}_{\mathrm{n}, \mathrm{m}} \mathrm{y}$ para cada $\mathrm{CD}_{\mathrm{n}}$.

Se empleó la escala Likert del 1 al 5 para los ítems medidos en escala ordinal, donde el valor 1 se correspondió con el criterio de muy bajo y el 5 con el de muy alto. Por otro lado, las variables ordinales $\mathrm{E}_{\mathrm{n}, \mathrm{m}}$ y $\mathrm{CD}_{\mathrm{n}}$ fueron calculadas para cada una de las empresas $i$. La variable $\mathrm{CS}_{\mathrm{i}}$ permitió identificar a las empresas más débiles y a las más fuertes dentro de la cadena. Se añadieron otras variables de sumo interés para el estudio, por ejemplo, el porcentaje de las ventas que se obtienen producto de la comercialización de camisetas $\left(\mathrm{G}_{1}\right)$ y el tamaño de la empresa $\left(\mathrm{G}_{2}\right)$. La variable $\mathrm{G}_{1}$ se determinó en 10 categorías con incrementos del $10 \%$ por cada una de ellas, lo que permitió caracterizar el porcentaje de representatividad de las ventas de camisetas respecto a las ventas totales desde el nivel más bajo (primera categoría menor o igual del 10\%) hasta el nivel más alto (última categoría mayor al 91\%). La variable $\mathrm{G}_{2}$ se categorizó según el criterio de la cantidad de personal ocupado $[39,40]$.

A partir de las variables ordinales $\mathrm{CD}_{\mathrm{n}}$ se formaron clústeres que agruparon a las empresas en cuanto al desempeño colaborativo dentro de la cadena de suministro. Para explorar el número de conglomerados se empleó, primeramente, un procedimiento jerárquico cuyos análisis se centraron en las tablas del Historial de Conglomeración obtenidas a partir de varias combinaciones de métodos de conglomeración con medidas de distancia. Se aplicó el "Método del Codazo" para detectar el mayor salto en los coeficientes de conglomeración, de manera tal que explore la cantidad de conglomerados a formar [41]. Posteriormente, se aplicó un procedimiento no jerárquico (conglomerado de $k$-medias) para determinar los conglomerados de pertenencia de cada una de las empresas analizadas [42].

Para probar relaciones entre las variables se emplearon los estadísticos Tau-b de Kendall y el de Spearman, como alternativas al coeficiente de Pearson para variables ordinales y con sus niveles de

Tabla 1. Formato general de la base de datos.

\begin{tabular}{|c|c|c|c|c|c|c|c|c|c|c|c|c|c|c|}
\hline \multirow{2}{*}{$\mathbf{i}$} & \multirow{2}{*}{$\mathbf{G}_{1}$} & \multirow{2}{*}{$\mathbf{G}_{2}$} & \multicolumn{4}{|c|}{$D_{1}$} & \multirow{2}{*}{$\mathrm{CD}_{1}$} & \multicolumn{4}{|c|}{ D7 } & \multirow[t]{2}{*}{$\mathrm{CD}_{7}$} & \multirow[t]{2}{*}{$\mathrm{CM}_{\mathrm{i}}$} & \multirow[t]{2}{*}{$\mathrm{CS}_{\mathrm{i}}$} \\
\hline & & & $\mathrm{E}_{1,1}$ & $\mathrm{E}_{1,2}$ & $\cdots$ & $\mathbf{E}_{1,1}$ & & $\mathbf{E}_{7,1}$ & $\mathbf{E}_{7,1}$ & $\ldots$ & $\mathbf{E}_{7,1}$ & & & \\
\hline 1 & & & & & & & & & & & & & & \\
\hline 2 & & & & & & & & & & & & & & \\
\hline$k$ & & & & & & & & & & & & & & \\
\hline Mo & & & & & & & $\mathrm{Mo}_{1}$ & & & & & $\mathrm{Mo}_{7}$ & & \\
\hline
\end{tabular}

Fuente: Autores. 
significación correspondientes [36]. Dichas relaciones pretendieron explicar, por un lado, las relaciones existentes entre las variables ordinales $\mathrm{CD}_{n}$, en aras de determinar si las empresas se desempeñan similarmente en todas ellas y, por otro lado, explicar si los clústeres formados lograban explicar algún tipo de relación del desempeño colaborativo (CS) con las variables porcentaje de las ventas que se obtienen producto de la comercialización de camisetas $\left(\mathrm{G}_{1}\right)$ y el tamaño de la empresa $\left(\mathrm{G}_{2}\right)$.

Finalmente se procedió a determinar el nivel de integración de la cadena mediante la siguiente expresión:

$$
N I_{c s}=\sum_{n=1}^{7} P_{n} * M o_{n}
$$

Donde:

- $P_{n}$ : Peso específico de importancia otorgada por los expertos para la dimensión $n$.

- $\quad N I_{c s}$ : Nivel de integración de la cadena.

- $M o_{n}$ : Moda calculada para cada $\mathrm{E}_{\mathrm{n}, \mathrm{m}} \mathrm{y}$ para cada $\mathrm{CD}_{\mathrm{n}}$.

Los decisores de los actores proporcionan el peso o grado de importancia a cada variable, mediante la Matriz de Proceso Analítico de Jerarquía, en relación a la cadena de estudio. Si el resultado alcanzado en $\mathrm{NI}_{\mathrm{CS}}$ es mayor que cero y menor o igual a uno, "Nivel Muy Bajo"; mayor que uno y menor o igual a dos, "Nivel Bajo"; mayor que dos y menor o igual a tres, "Nivel Medio", mayor que tres y menor o igual a cuatro, "Nivel Alto" y mayor que cuatro y menor o igual a cinco, "Nivel Muy Alto". Estos cinco estados para $\mathrm{NI}_{\mathrm{CS}}$ contribuyeron con el análisis de que si las dimensiones son bien evaluadas es porque la ICS ha alcanzado un nivel adecuado. Si están bien, se harán todos los esfuerzos posibles para lograr la excelencia pasando por un estado de muy bien, lo que implica alcanzar niveles superiores en el desempeño de las dimensiones. Por otro lado, se corre el riesgo de que las dimensiones presenten algún grado de deterioro, por lo que se hace necesario establecer una categoría de regular, que representa la antesala de la peor de las categorías a asignar, que es la de mal, e implica el deterioro total del $\mathrm{NI}_{\mathrm{CS}}$.

La determinación del nivel de integración de la cadena de suministro está en función del actor de más bajo valor de NPC en la cadena. Esto se cumple siempre y cuando el actor seleccionado presenta relación directa con el producto y servicio final de la cadena. A partir de esto, se concluye con el cálculo del nivel de integración, y a partir de este resultado se definen las estrategias y los objetivos conjuntos que debe seguir la cadena. Para ello se utiliza la matriz de selección de estrategias de integración en las cadenas de suministro [18].

\section{RESULTADOS}

La cadena de suministros de la industria textil en Ecuador consta de 109 actores, a pesar del alto número de actores analizados no representa el total de la población, de ellas 96 se dedican a la confección de camisetas dentro de su variedad de productos. Dicha muestra representa el $88 \%$ del total de empresas que conforman la presente investigación, ofreciendo un nivel de confianza del $96 \%$ para un error estándar del $5 \%$. La muestra fue calculada a través de la fórmula de tamaño de la muestra para población finita. La cadena de suministro en estudio se divide en cinco eslabones: proveedores, transformadores, confección, vendedores y clientes (Figura 2).

Según los valores obtenidos para el Alpha de Cronbach, las dimensiones del estudio: Estrategia $\left(D_{1}\right)$, Información $\left(D_{2}\right)$, Planeación $\left(D_{3}\right)$ y Compra $\left(\mathrm{D}_{4}\right)$ mostraron valores de consistencia buenos, superiores al 0,8 , mientras que las dimensiones Inventario $\left(D_{5}\right)$, Transporte $\left(D_{6}\right)$ y Desempeño $\left(D_{7}\right)$ resultaron con valores superiores a 0,7 por lo que fueron evaluadas como aceptables (Tabla 2).

Además, se observó que los resultados del Alpha de Cronbach no mejorarían significativamente si se decidiera eliminar algún elemento de los considerados dentro de las dimensiones, destacando el carácter de unidimensionalidad.

\section{Análisis descriptivo por elementos}

De la muestra analizada, el 82,24\% (88 empresas) se dedican a la confección de camisetas, lo cual reafirma la importancia de este estudio para las industrias textiles de la zona. De ellas, el 54,5\% (48 empresas) y el 45,5\% (40 empresas) se clasificaron como pequeñas y medianas empresas, respectivamente (variable $\mathrm{G}_{2}$ ). Al observar el porcentaje que representan las ventas de camisetas dentro de las ventas totales (variable $\mathrm{G}_{1}$ ), se observó que la cadena posee dos grandes grupos de empresas con medianas que se 
Fuente: Modificado de Orozco et. al [43].

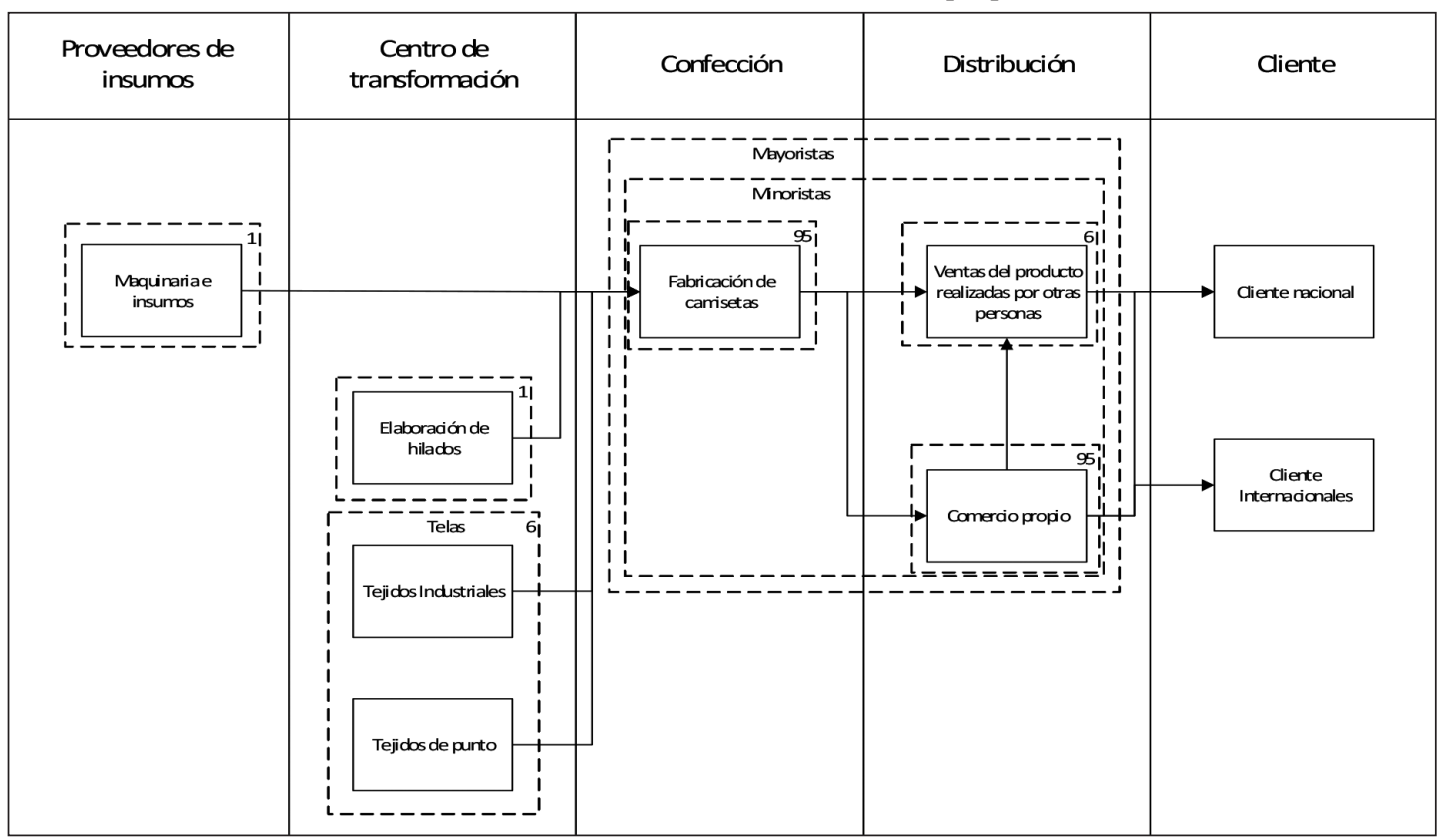

Figura 2. Cadena de suministro de camisetas del Ecuador.

Tabla 2. Valores del Alpha de Cronbach.

\begin{tabular}{|c|c|c|c|c|c|c|c|c|c|c|c|c|c|c|}
\hline \multirow{2}{*}{ Dimemsión } & \multirow{2}{*}{$\begin{array}{c}\text { Alfa de } \\
\text { Cronbach }\end{array}$} & \multicolumn{13}{|c|}{ Alfa de Cronbach si se elimina el elemento } \\
\hline & & $E_{n, 1}$ & $\mathbf{E}_{\mathbf{n}, 2}$ & $\mathbf{E}_{n, 3}$ & $\mathbf{E}_{\mathbf{n}, \mathbf{4}}$ & $\mathbf{E}_{\mathbf{n}, \mathbf{5}}$ & $E_{n, 6}$ & $\mathbf{E}_{\mathbf{n}, 7}$ & $\mathbf{E}_{\mathbf{n}, 8}$ & $E_{n, 9}$ & $E_{n, 10}$ & $E_{n, 11}$ & $E_{n, 12}$ & $E_{n, 13}$ \\
\hline Estrategia $\left(\mathrm{D}_{1}\right)$ & 0,88 & 0,86 & 0,86 & 0,87 & 0,87 & 0,87 & 0,86 & 0,86 & 0,88 & 0,89 & & & & \\
\hline Información $\left(\mathrm{D}_{2}\right)$ & 0,85 & 0,82 & 0,82 & 0,83 & 0,83 & 0,84 & 0,85 & 0,82 & 0,84 & & & & & \\
\hline Planeación $\left(\mathrm{D}_{3}\right)$ & 0,84 & 0,81 & 0,81 & 0,83 & 0,82 & 0,77 & 0,84 & 0,82 & 0,83 & & & & & \\
\hline Compra $\left(\mathrm{D}_{4}\right)$ & 0,85 & 0,84 & 0,84 & 0,84 & 0,85 & 0,86 & 0,84 & 0,84 & 0,84 & 0,84 & 0,84 & 0,85 & 0,84 & 0,85 \\
\hline Inventario $\left(\mathrm{D}_{5}\right)$ & 0,79 & 0,75 & 0,78 & 0,79 & 0,76 & 0,76 & 0,76 & 0,76 & 0,77 & & & & & \\
\hline Transporte $\left(\mathrm{D}_{6}\right)$ & 0,73 & 0,73 & 0,69 & 0,69 & 0,71 & 0,74 & 0,71 & 0,70 & 0,70 & 0,71 & 0,74 & 0,73 & 0,70 & \\
\hline Desempeño $\left(\mathrm{D}_{7}\right)$ & 0,78 & 0,71 & 0,72 & 0,72 & 0,78 & 0,72 & 0,74 & & & & & & & \\
\hline
\end{tabular}

Fuente: Autores.

localizan en los rangos del 21-30\% (categoría 3) y del $71-80 \%$ de las ventas (categoría 8) (ver a continuación las Figuras 2 y 3).

El resumen de las frecuencias relativas de todas las dimensiones es el que se muestra en la Tabla 3.

- Dimensión Estrategia $\left(\mathbf{D}_{1}\right)$ : En el diseño de la estrategia, el 52,27\% de las empresas de la cadena consideraron a niveles muy bajos a sus proveedores y el $62,50 \%$ a niveles bajos a sus clientes. Sin embargo, el 77,30\% de las empresas plantearon que colegian su estrategia con los clientes, mientras que el resto colegian su estrategia con los proveedores y otros integrantes de la cadena. El 52,27\% valoró que la estrategia es colaborativa a niveles bajos, mientras que el 35,23\% esta colaboración la valoró a muy bajo nivel. El 62,50\% consideró que debe ser colaborativa con los clientes, el $18,20 \%$ con los proveedores y el 19,30\% dentro de la misma entidad. La medida en que los objetivos estratégicos consideraron a los proveedores se comportó a niveles bajos y 
Fuente: Autores.

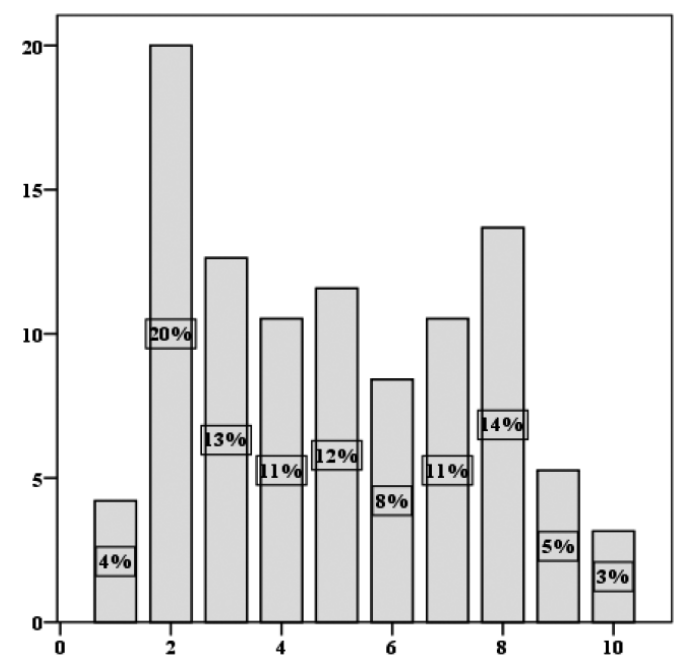

Figura 3. Histograma de frecuencias absolutas por categoría de la variable $\mathrm{G}_{1}$.

muy bajos con un $87,50 \%$. Este mismo aspecto, pero desde la perspectiva de los clientes, se comportó a un nivel bajo a un $57,95 \%$. En cuanto a la formulación de los escenarios las empresas consideraron a sus proveedores y clientes a un nivel bajo con una frecuencia de $46,59 \%$ y $42,05 \%$, respectivamente; el $95,45 \%$ consideraron al mercado nacional, pero a niveles que oscilan entre muy bajos y bajos; y el 87,50\% consideraron al mercado internacional a un nivel muy bajo.

- Dimensión Información ( $\left.\mathbf{(}_{\mathbf{2}}\right)$ : El 47,73\% de las empresas planteó que firman sus contratos con diferentes entidades en una baja expresión, el $89,78 \%$ y el $92,04 \%$ plantearon que elaboran sus contratos de forma colaborativa con sus proveedores y clientes, aunque esto se manifiesta en los niveles bajos y muy bajos, respectivamente. El 61,40\% planteó que en sus contratos de compra si elaboran una cláusula para la seguridad y confiabilidad de la información que se brinda a los proveedores mientras que el 51,10\% planteó que también elaboran dicha cláusula para la información que brindan a otras entidades. El 62,50\% planteó que en sus contratos de venta si elaboran una cláusula para la seguridad y confiabilidad de la información que se brinda a los clientes. El 59,09\% planteó que esta información que se brinda dentro de la

Tabla 3. Valores de las frecuencias relativas para $E_{n, m}$.

\begin{tabular}{|c|c|c|c|c|c|c|c|c|c|c|c|c|c|c|}
\hline $\mathbf{D}_{\mathrm{i}}$ & Categoría & $E_{n, 1}$ & $E_{n, 2}$ & $\mathbf{E}_{n, 3}$ & $E_{n, 4}$ & $E_{n, 5}$ & $E_{n, 6}$ & $\mathbf{E}_{\mathbf{n}, 7}$ & $\mathbf{E}_{n, 8}$ & $E_{n, 9}$ & $E_{n, 10}$ & $E_{n, 11}$ & $E_{n, 12}$ & $E_{n, 13}$ \\
\hline \multirow{3}{*}{$\begin{array}{c}\text { Estrategia } \\
\qquad\left(D_{1}\right)\end{array}$} & Muy bajo & 52,3 & 15,9 & 35,2 & 44,3 & 25,0 & 36,4 & 37,5 & 46,6 & 87,5 & & & & \\
\hline & Bajo & 43,2 & 62,5 & 52,3 & 43,2 & 58,0 & 46,6 & 42,0 & 48,9 & 12,5 & & & & \\
\hline & Medio & 4,0 & 21,6 & 12,5 & 12,5 & 17,0 & 17,0 & 20,5 & 4,5 & 0,0 & & & & \\
\hline \multirow{3}{*}{$\begin{array}{c}\text { Información } \\
\left(\mathrm{D}_{2}\right)\end{array}$} & Muy bajo & 37,5 & 47,7 & 43,2 & 28,4 & 37,5 & 78,4 & 35,2 & 28,4 & & & & & \\
\hline & Bajo & 47,7 & 42,0 & 48,9 & 59,1 & 55,7 & 15,9 & 47,7 & 53,4 & & & & & \\
\hline & Medio & 14,8 & 10,2 & 8,0 & 12,5 & 6,8 & 5,7 & 17,0 & 18,2 & & & & & \\
\hline \multirow{3}{*}{$\begin{array}{c}\text { Planeación } \\
\left(D_{3}\right)\end{array}$} & Muy bajo & 3,4 & 25,0 & 20,5 & 18,2 & 14,5 & 29,5 & 44,3 & 19,3 & & & & & \\
\hline & Bajo & 68,2 & 52,3 & 58,0 & 67,0 & 37,5 & 61,4 & 48,9 & 58,0 & & & & & \\
\hline & Medio & 28,4 & 22,7 & 21,6 & 14,8 & 47,7 & 9,1 & 6,8 & 22,7 & & & & & \\
\hline \multirow{3}{*}{$\begin{array}{c}\text { Compra } \\
\left(\mathrm{D}_{4}\right)\end{array}$} & Muy bajo & 46,6 & 45,5 & 42,0 & 44,3 & 77,3 & 50,0 & 35,2 & 38,6 & & & & & \\
\hline & Bajo & 42,0 & 45,5 & 47,7 & 51,1 & 22,7 & 40,9 & 50,0 & 52,3 & & & & & \\
\hline & Medio & 11,4 & 9,1 & 10,2 & 4,5 & 0,0 & 9,1 & 14,8 & 9,1 & 8,0 & 6,8 & 6,8 & 6,8 & 5,7 \\
\hline \multirow{3}{*}{$\begin{array}{c}\text { Inventario } \\
\left(D_{5}\right)\end{array}$} & Muy bajo & 35,2 & 38,6 & 44,3 & 37,5 & 43,2 & 34,1 & 38,6 & 35,2 & & & & & \\
\hline & Bajo & 46,6 & 44,3 & 52,3 & 52,3 & 48,9 & 56,8 & 52,3 & 55,7 & & & & & \\
\hline & Medio & 18,2 & 17,0 & 3,4 & 10,2 & 8,0 & 9,1 & 9,1 & 9,1 & & & & & \\
\hline \multirow{3}{*}{$\begin{array}{c}\text { Transporte } \\
\left(D_{6}\right)\end{array}$} & Muy bajo & 3,4 & 37,5 & 39,8 & 39,8 & 14,8 & 22,7 & 55,7 & 68,2 & 21,6 & 75,0 & 21,6 & 34,1 & \\
\hline & Bajo & 34,1 & 29,5 & 37,5 & 30,7 & 39,8 & 27,3 & 26,1 & 19,3 & 30,7 & 12,5 & 43,2 & 38,6 & \\
\hline & Medio & 62,5 & 33,0 & 22,7 & 29,5 & 45,5 & 48,9 & 18,2 & 12,5 & 47,7 & 12,5 & 34,1 & 27,3 & \\
\hline \multirow{3}{*}{$\begin{array}{c}\text { Desempeño } \\
\left(\mathrm{D}_{7}\right)\end{array}$} & Muy bajo & 54,5 & 52,3 & 47,7 & 48,9 & 47,7 & 43,2 & & & & & & & \\
\hline & Bajo & 35,5 & 38,6 & 43,2 & 51,1 & 40,9 & 48,9 & & & & & & & \\
\hline & Medio & 10,2 & 9,1 & 9,1 & 0,0 & 11,4 & 8,0 & & & & & & & \\
\hline
\end{tabular}

Fuente: Autores. 
Fuente: Autores.

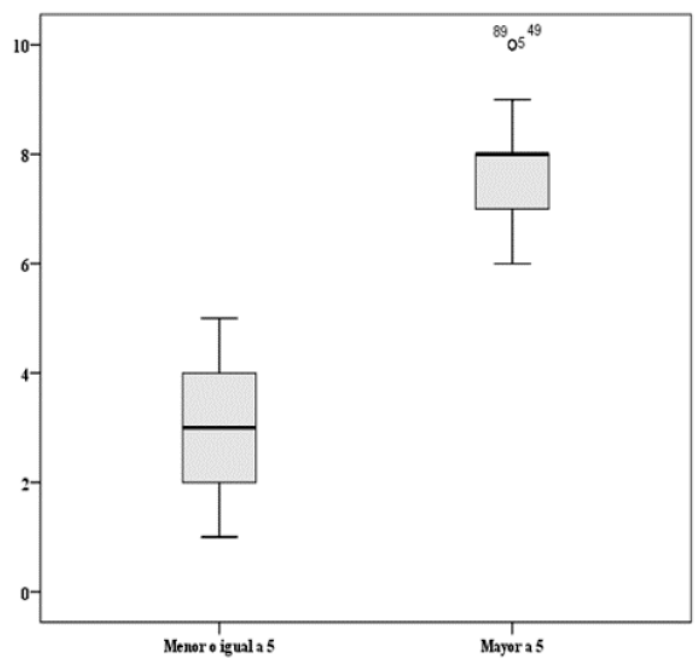

Figura 4. Diagrama de cajas para la variable $\mathrm{G}_{1}{ }^{7}$.

cadena si fomenta las relaciones de colaboración, pero a niveles bajos y el 55,68\% de los casos calificó la retroalimentación a los proveedores a un nivel bajo. El 65,9\% de los casos planteó que la información no es gestionada por una empresa focal dentro de la cadena y el 78,41\% que el nivel de la información con que cuentan de toda la cadena se encuentra a nivel muy bajo. El $47,73 \%$ y $53,41 \%$ plantearon que la información operativa de la logística de la entidad es accesible a los proveedores y a los clientes a un nivel bajo, respectivamente.

- Dimensión Planeación $\left(\mathbf{D}_{3}\right)$ : En el proceso de planificación se consideraron a los clientes finales de la cadena a un nivel bajo, dado que el 68,18\% de las empresas opinaron de esta manera. El horizonte de planificación resultó a corto y muy corto plazos ya que supera el trimestre sólo en el $35,2 \%$ de las empresas, en el resto predomina la planificación semanal y mensual. El 52,27\%, el $57,95 \%$ y el $67,05 \%$ apreciaron que sus planes tienen en cuenta la característica de sus clientes, de sus proveedores y de la competencia a un nivel bajo, respectivamente; predominando el $47,73 \%$ de los planes tuvieron en cuenta las

7 Para su obtención se determinó a $\mathrm{G}_{1}$ en dos categorías: Menor o igual a 5 que agrupó a las empresas con porcentajes de ventas de camisetas por debajo del $50 \%$ y Mayor a 5 que agrupó al resto de las empresas con porcentajes superiores al 50\%. características de la propia entidad a un nivel medio. El $61,36 \%$ y el $48,86 \%$ de los casos se caracterizó por niveles bajos de correlación existente entre la planificación de la demanda de la empresa y la de sus proveedores y clientes. A lo que el 57,95\% afirmó que los pronósticos de la demanda que realizan se corresponden a un nivel bajo con las necesidades de sus clientes.

- Dimensión Compra $\left(\mathbf{D}_{4}\right)$ : El 88,64\%, el 90,90\%, el $89,78 \%$ y el $95,46 \%$ evaluó a niveles bajos y muy bajos que sus planes de compras se fundamentan en: el balance de mercancías, las compras históricas, las campañas de ventas y la experiencia del planificador, respectivamente. El $77,27 \%$ consideraron a niveles muy bajos que fundamentan su plan de compra sobre la base de otras fuentes. Es de destacar como las empresas consideraron a niveles entre muy bajos y bajos la medida en que las compras que realizan consideran las necesidades de los clientes $(50,00 \%)$, el comportamiento de los inventarios $(50,00 \%)$, las sugerencias de la propia entidad $(52,27 \%)$, los descuentos por cantidad de los proveedores (46.59\%), el plan de demanda $(52,27 \%)$, las capacidades de los proveedores $(57,95 \%)$, los ciclos de la cadena de suministro para el reaprovisionamiento $(47,73 \%)$ y los ciclos para el producto terminado $(50,00 \%)$.

- Dimensión Inventario $\left(\mathbf{D}_{5}\right)$ : Existió un predominio del desconocimiento de las empresas respecto a los parámetros de los inventarios que manejan los proveedores y de los clientes, con una frecuencia de 46,59\% y 44,32\%, respectivamente. A lo que se añade que, en cuanto a inventarios se refiere, los proveedores presentan un nivel bajo de colaboración con la entidad (52,27\%), bajas alianzas estratégicas con la entidad $(52,27 \%)$ y que poco conocen de lo que piensan los clientes finales de la cadena (48,86\%). Un aproximado del 56,82\% de las empresas manifestaron a un nivel bajo que conocen la ubicación de sus inventarios dentro de la cadena de suministros por medios propios, por los proveedores y por los clientes.

- Dimensión Transporte $\left(\mathbf{D}_{6}\right)$ : En la distribución de los productos terminados a los clientes existió un predominio de los pedidos planificados $(62,50 \%)$ y de las solicitudes urgentes de los clientes $(45,45 \%)$, ambas variantes evaluadas a un nivel medio. El resto, como fueron las de 
seguir un programa de distribución, un ritmo estable de distribución y el comportamiento de los inventarios de los clientes, fueron evaluadas compartiendo los niveles muy bajos y bajos. Las rutas de la distribución para la mercancía se realizan mayormente sobre la base de la experiencia de los conductores $(48,86 \%)$ y de las condiciones físicas de las rutas $(47,73 \%)$, ambas evaluadas a un nivel medio. Apenas existieron otras alternativas en el diseño de este ruteo, como por ejemplo los esquemas fijos de rutas o la búsqueda de soluciones óptimas para la distribución. En cuanto el empleo de los medios de transporte para la distribución de las mercancías, se colabora más con los clientes que con los proveedores para frecuencias de $64,77 \%$ y $72,73 \%$, respectivamente, pero a niveles muy bajos y bajos, lo que denota cierta subutilización de los medios de transporte.

- Dimensión Desempeño $\left(\mathbf{D}_{7}\right)$ : El 89,78\% de las empresas denotaron un predominio de indicadores muy básicos para evaluar su propio desempeño, mas no el desempeño de sus clientes y de sus proveedores. Los clientes son de interés más para la propia entidad que para los proveedores de esta a niveles medio $(51,1 \%)$ y bajo $(40,9 \%)$, respectivamente.

Los valores estimados en el análisis de correlación para los estadísticos Tau_b de Kendall y Rho de Spearman destacaron relaciones positivas que fueron evaluadas entre significativas y moderadas al comportarse superiores a 0,5 y por debajo de 0,84 (ver Tabla 4). Las relaciones significativas, se ubican entre: las estrategias y el inventario $(0,702$ b), la información y las compras $(0,742 \mathrm{~b})$, las compras y el inventario $(0,816 \mathrm{~b})$, y las compras y el desempeño $(0,731 \mathrm{~b})$. En la segunda prueba los La excepción estuvo en la dimensión Transporte $\left(\mathrm{CD}_{6}\right)$ que correlacionó muy débil con el resto de las dimensiones, aun así, se decidió mantener para los análisis posteriores.

\section{Clasificación por conglomerado y estrategias de integración}

El método jerárquico aplicado para diferentes combinaciones de métodos de conglomeración y medidas de distancia arrojaron los coeficientes de conglomeración de la Tabla 5, en la que se observa que el mayor salto en los coeficientes de conglomeración ocurrió entre las etapas 86 y 87, sugiriendo la formación de dos conglomerados.

El procedimiento no jerárquico (conglomerado de $k$-medias) formó un primer conglomerado al que pertenecieron 74 empresas evaluadas a un nivel muy bajo en casi todas las dimensiones y un segundo conglomerado en el que 14 empresas se evaluaron a un nivel bajo y medio y donde la mayor parte de estas fueron empresas de tamaño mediano (ver Tablas 6 y 7). Es de destacar cómo la dimensión Transporte $\left(\mathrm{CD}_{6}\right)$ no contribuyó sobremanera a discriminar la formación de estos grupos, arrojando el mismo centro final para ambos conglomerados con una avaluación de nivel bajo.

El conglomerado de pertenencia obtenido mediante el procedimiento no jerárquico (conglomerado de $\mathrm{k}$-medias) fue guardado en la base de datos en la variable conglomerado de pertenencia $(\mathrm{CM})$ de la que se aprovechó su información ordinal y se correlacionó con las variables porcentaje de producción de camisetas $\left(\mathrm{G}_{1}\right)$, tamaño de la empresa $\left(\mathrm{G}_{2}\right)$ y puntuación total de colaboración (CS) (ver Tabla 8). Esto permitió validar los resultados obtenidos con la formación de los conglomerados. La relación fuerte y positiva $(0,947$ y 0,960$)$ que se obtuvo entre CM y

Tabla 4. Coeficientes de correlación para las CDn.

\begin{tabular}{|c|c|c|c|c|c|c|c|c|c|c|c|c|c|c|}
\hline & \multicolumn{2}{|c|}{$\mathrm{CD}_{1}$} & \multicolumn{2}{|c|}{$\mathrm{CD}_{2}$} & \multicolumn{2}{|c|}{$\mathrm{CD}_{3}$} & \multicolumn{2}{|c|}{$\mathrm{CD}_{4}$} & \multicolumn{2}{|c|}{$\mathrm{CD}_{5}$} & \multicolumn{2}{|c|}{$\mathrm{CD}_{6}$} & \multicolumn{2}{|c|}{$\mathrm{CD}_{7}$} \\
\hline $\mathrm{CD}_{1}$ & $1,00^{\mathrm{a}}$ & $1,00^{b}$ & $0,577^{* * *}$ & $0,587^{* * *}$ & $0,642^{* * *}$ & $0,665^{* *}$ & $0,674^{* * *}$ & 0,686 *** & $0,682^{* *}$ & $0,707 * *$ & $0,206^{*}$ & $0,211^{*}$ & $0,551^{* * *}$ & $0,574^{* * *}$ \\
\hline $\mathrm{CD}_{2}$ & $0,577^{* * *}$ & 0,587 *** & 1,00 & 1,00 & $0,622^{* *}$ & 0,647 & $0,719^{* * *}$ & $0,742^{* * *}$ & $0,642^{* * *}$ & 0,66 & 0,062 & 0,065 & $0,642 * *$ & $0,673^{* *}$ \\
\hline $\mathrm{CD}_{3}$ & $0,642 * *$ & $665^{* *}$ & 0,622 & $0,647 * *$ & 1,00 & 1,00 & $0,566^{* * *}$ & $0,593^{* * *}$ & $0,593^{* * *}$ & 0,620 *** & $0,215^{*}$ & $0,227 *$ & $0,528 * *$ & $0,561 * *$ \\
\hline $\mathrm{CD}_{4}$ & $0,674 * *$ & $86 * *$ & $0,719 * *$ & 742 & 0,56 & & 1,00 & & $0,794^{* *}$ & $0,816^{* * *}$ & 0,112 & 0,114 & $0,702^{* * *}$ & $0,731^{* * *}$ \\
\hline $\mathrm{CD}_{5}$ & $0,682^{* * *}$ & $707 * *$ & $0,642 * *$ & $0,665^{* *}$ & $0,593^{* *}$ & $0,620^{* *}$ & $0,794^{* * *}$ & $0,819^{* * *}$ & 1,00 & $\overline{1,00}$ & 0,145 & 0,152 & $0,530^{* *}$ & $0,572^{* *}$ \\
\hline $\mathrm{CD}_{6}$ & $206^{*}$ & & 0,062 & 0,065 & $0,215^{* *}$ & $0,227^{*}$ & 0,112 & & 0,145 & & 1,00 & 1,00 & 0,065 & 0,068 \\
\hline $\mathrm{CD}_{7}$ & $551^{* * *}$ & $0,574 * *$ & $0,642 * *$ & $0,673^{* * *}$ & $0,528 * *$ & $0,561 * *$ & $0,702^{* *}$ & $0,731^{* * *}$ & 0,530 *** & $0,572 * *$ & 0,065 & 0,68 & 1,00 & 1,00 \\
\hline
\end{tabular}

Fuente: Autores. a: Tau_b de Kendall. b: Rho de Spearman. ** La correlación es significativa al nivel 0,01 (bilateral). * La correlación es significativa al nivel 0,05 (bilateral). 
Tabla 5. Resultados de las iteraciones con el método jerárquico.

\begin{tabular}{|c|c|c|}
\hline \multicolumn{3}{|c|}{ Historia de conglomeración } \\
\hline Etapa & Coeficientes $^{\mathbf{a}}$ & Coeficientes $^{\mathbf{b}}$ \\
\hline 1 & 0 & 0 \\
- & - & - \\
- & - & - \\
\hline 85 & 3,55 & 3,166 \\
\hline 86 & 4,077 & 3,625 \\
\hline 86 & 12,208 & 8,106 \\
\hline
\end{tabular}

a: coeficientes de conglomeración para el método de vinculación promedio (intergrupos) con medida distancia euclídea al cuadrado.

b: coeficientes de conglomeración para el método de conglomeración vinculación promedio (Inter-grupos) con medida de distancia de bloque.

Fuente: Autores.

Tabla 6. Centros de los conglomerados finales.

\begin{tabular}{|l|l|l|}
\hline \multicolumn{3}{|c|}{ Centros de los conglomerados finales } \\
\hline & \multicolumn{2}{|c|}{ Conglomerados } \\
\hline $\mathrm{CD}_{1}$ & 1 & 2 \\
\hline $\mathrm{CD}_{2}$ & 1 & 2 \\
\hline $\mathrm{CD}_{3}$ & 1 & 3 \\
\hline $\mathrm{CD}_{4}$ & 1 & 2 \\
\hline $\mathrm{CD}_{5}$ & 1 & 3 \\
\hline $\mathrm{CD}_{6}$ & 2 & 2 \\
\hline $\mathrm{CD}_{7}$ & 1 & 3 \\
\hline
\end{tabular}

Fuente: Autores.

Tabla 7. Cantidad de empresas por conglomerado.

\begin{tabular}{|l|c|c|}
\hline \multicolumn{2}{|c|}{ Número de casos en cada conglomerado } \\
\hline \multirow{2}{*}{ Conglomerado } & 1 & 74 \\
\cline { 2 - 3 } & 2 & 14 \\
\hline Válidos & & 88 \\
\hline Perdidos & & 0 \\
\hline
\end{tabular}

Fuente: Autores.
CS corroboró el análisis realizado en la formación de los conglomerados, en el que se dijo que el conglomerado 1 se compuso por empresas evaluadas a un nivel muy bajo, mientras que el conglomerado 2 se formó con empresas evaluadas a un nivel bajo y medio de colaboración. Posteriormente, se destaca una relación positiva pero débil $(0,227$ y 0,227$)$ entre $\mathrm{CM} \mathrm{y}_{2}$, lo que permitió afirmar que por lo general las empresas clasificadas como medianas se ubicaron en el conglomerado 2 y poseen un nivel de desempeño colaborativo más alto.

Como se puede observar en la Tabla 9, la evaluación del nivel de integración en la cadena de camisetas es de 1,4.

Objetivos y estrategias para la mejora de la Integración de la cadena de suministros

A partir de estos resultados, se puede concluir que la cadena de suministro en estudio presenta un nivel de integración bajo, y se ubica en la etapa de "Negociaciones de mercado" (ver sección 2. La integración en la cadena de suministro). Las estrategias en esta etapa resultan: Discusión enfocada a liderazgos en costos; diferenciación; o enfoque en nicho. En este sentido, los objetivos conjuntos se centran en la satisfacción de los clientes:

- Elevar el nivel de integración y con ello la mejora de la competitividad de la cadena.

- Aumentar la variedad de los productos textiles para la influencia en el nivel de servicio.

- Aumentar el valor agregado a las necesidades de los clientes a través de telas más preferidas por el cliente y formas de fabricación con la meta de incrementos en el valor de uso de las camisetas.

Como resultado de las debilidades que se identifican y el bajo estado de integración entre los actores de la cadena textil, se hace necesario plantear estrategias de mejora que contribuyan a disminuir

Tabla 8. Coeficientes de correlación entre variables.

\begin{tabular}{|l|c|l|l|l|l|l|l|l|}
\cline { 2 - 9 } \multicolumn{1}{c|}{} & \multicolumn{2}{c|}{$\mathbf{G}_{\mathbf{1}}$} & \multicolumn{2}{c|}{$\mathbf{G}_{\mathbf{2}}$} & \multicolumn{2}{c|}{ CM } & \multicolumn{2}{c|}{ CS } \\
\hline $\mathrm{G}_{1}$ & $1^{\mathrm{a}}$ & $1^{\mathrm{b}}$ & 0,16 & 0,185 & 0,019 & 0,022 & $-0,008$ & $-0,009$ \\
\hline $\mathrm{G}_{2}$ & 0,16 & 0,185 & 1 & 1 & $0,227^{*}$ & $0,227^{*}$ & 0,2 & 0,203 \\
\hline $\mathrm{CM}$ & 0,019 & 0,022 & $0,227^{*}$ & $0,227^{*}$ & 1 & 1 & $0,947^{* *}$ & $0,960^{* *}$ \\
\hline $\mathrm{CS}$ & $-0,008$ & $-0,009$ & 0,2 & 0,203 & $0,947^{* *}$ & $0,960^{* *}$ & 1 & 1 \\
\hline
\end{tabular}

a: Tau_b de Kendall. b: Rho de Spearman.

** La correlación es significativa al nivel 0,01 (bilateral).

* La correlación es significativa al nivel 0,05 (bilateral.

Fuente: Autores. 
Tabla 9. Resultados del nivel de integración.

\begin{tabular}{|l|c|c|c|c|}
\hline \multicolumn{1}{|c|}{ Dimensión $\mathbf{D}_{\mathbf{n}}$} & $\mathbf{M o}_{\mathbf{n}}$ & Evaluación $\mathbf{D}_{\mathbf{n}}$ & $\mathbf{P}_{\mathbf{n}}$ & $\mathbf{P}_{\mathbf{n}} * \mathbf{M o}_{\mathbf{n}}$ \\
\hline Estrategia $\left(\mathrm{D}_{1}\right)$ & 1 & Muy bajo & 0,10 & 0,10 \\
\hline Información $\left(\mathrm{D}_{2}\right)$ & 1 & Muy bajo & 0,10 & 0,10 \\
\hline Planeación $\left(\mathrm{D}_{3}\right)$ & 2 & bajo & 0,30 & 0,60 \\
\hline Compras $\left(\mathrm{D}_{4}\right)$ & 1 & Muy bajo & 0,10 & 0,10 \\
\hline Inventario $\left(\mathrm{D}_{5}\right)$ & 1 & Muy bajo & 0,10 & 0,10 \\
\hline Transporte $\left(\mathrm{D}_{6}\right)$ & 2 & bajo & 0,10 & 0,20 \\
\hline Desempeño $\left(\mathrm{D}_{7}\right)$ & 1 & Muy bajo & 0,20 & 0,20 \\
\hline \multicolumn{4}{|c|}{$\mathrm{NI}_{C S}=\sum_{n=1}^{7} P_{n} * \mathbf{M o}_{n}$} & 1,40 \\
\hline
\end{tabular}

Fuente: Autores.

estas brechas. Dentro de las estrategias que pueden implementar, se destacan:

- Definir políticas y estrategias entre los actores que conforman los diferentes eslabones y a la vez que fomenten las mejores relaciones entre los clientes y proveedores.

- Incluir dentro de los objetivos estratégicos a los clientes, por ser parte de la sostenibilidad de la cadena y de cada negocio en particular.

- Definir técnicas que posibiliten la definición de los escenarios en donde intervengan los clientes y proveedores como los decisores por excelente.

- Establecer contratos específicos y marcos entre los actores, para la definición de indicadores y variables de las negociaciones junto con cláusulas para la seguridad y confiabilidad de la información.

- Diseñar un sistema de información que posibilite la interrelación de la información entre los actores (proveedores - clientes), donde se tenga acceso a la información operativa de la logística.

- Estimar la demanda de productos textiles en toda la cadena donde se facilite la toma de decisiones a corta, mediano y largo plazo.

- Establecer mecanismos de colaboración que permitan la elaboración de la planificación estratégica de la cadena, y así fortalecer la planeación a largo plazo.

- Diseñar productos creativos e innovadores que permitan la posición de los productos en el mercado, y se correspondan en mayor medida con los que ofrece la competencia.

- Establecer planes de compras más ajustados a las ventas y a los datos históricos, con el fin de disminuir los bajos niveles de rotación del inventario, los ciclos y ajustar las necesidades de los clientes a las compras realizadas a los proveedores.

- Definir una política de compra que potencie los descuentos y las compras unificadas entre los actores para aumentar volumen y disminuir los precios.

- Potenciar las alianzas estratégicas entre socios, proveedores y clientes que facilite la colaboración en los inventarios, elemento básico del endeudamiento de estos actores,

- Potenciar la creación de operadores logísticos que distribuyan y transporten los pedidos de los actores hacia los clientes, y la materia prima propiciando una mejor optimización de las rutas y del volumen de transportación, y por ende mejora en los costos logísticos.

- Calcular la capacidad productiva para la determinación del cuello de botella limitante de la cadena.

- Establecer indicadores en la cadena que faciliten la evaluación del desempeño y la mejora.

- Elegir el actor focal de la cadena, realizar análisis financieros a cada actor de la cadena para reinversión del capital.

- Analizar las compras y la evaluación de los proveedores nacionales e internacionales.

- Diagnosticar el sistema de los proveedores y los operadores logísticos de la cadena y;

- Formar al personal en base a las competencias necesarias para la gestión en la cadena de suministro descritas en la Tabla 1.

Asimismo, en la Tabla 10 se propone estimular el desarrollo de la cadena de suministro mediante la identificación y puesta en marcha de acciones que contrarresten algunos problemas identificados. 
Tabla 10. Propuestas de acciones para impulsar el desarrollo de la cadena textil en Ecuador.

\begin{tabular}{|c|c|}
\hline Problemas & ¿Qué investigar? \\
\hline $\begin{array}{l}\text { Baja inserción de proveedores y clientes en la } \\
\text { estrategia, objetivos y escenarios conjuntos de } \\
\text { la cadena. }\end{array}$ & $\begin{array}{l}\text { Identificar y trabajar en la coordinación de estrategia, objetivos } \\
\text { y escenarios de la cadena que aseguren la planeación conjunta. }\end{array}$ \\
\hline $\begin{array}{l}\text { Poca inclusión del mercado internacional en los } \\
\text { elementos estratégicos de la cadena. }\end{array}$ & $\begin{array}{l}\text { Realizar benchmarking en el mercado internacional, para la selección } \\
\text { de productos estrellas y la adecuación de las mejores prácticas. }\end{array}$ \\
\hline $\begin{array}{l}\text { Baja capacitación y formación del potencial humano } \\
\text { que pertenecen a los actores de la cadena. }\end{array}$ & $\begin{array}{l}\text { Incrementar la oferta de cursos de capacitación en las universidades } \\
\text { y entidades del territorio. }\end{array}$ \\
\hline $\begin{array}{l}\text { Poca utilización de contratos específicos y marcos } \\
\text { entre los actores para las negociaciones conjuntas } \\
\text { de la cadena }\end{array}$ & $\begin{array}{l}\text { Capacitar a los actores de la cadena, de la utilidad del contrato } \\
\text { para el correcto funcionamiento del negocio. }\end{array}$ \\
\hline $\begin{array}{l}\text { Inexistencia de cláusulas para la seguridad y } \\
\text { confiabilidad de la información. }\end{array}$ & $\begin{array}{l}\text { Establecer formatos de contratos, que cuenten con cláusulas de } \\
\text { seguridad y confiabilidad de la información. }\end{array}$ \\
\hline $\begin{array}{l}\text { Ineficiente sistema de información entre los actores, } \\
\text { donde se tenga acceso a la información operativa } \\
\text { de la logística. }\end{array}$ & $\begin{array}{l}\text { Diseñar un sistema de información entre todos los actores, accesible } \\
\text { y simple que permita la toma de decisiones. }\end{array}$ \\
\hline $\begin{array}{l}\text { Poca estimación de la demanda de productos textiles } \\
\text { en toda la cadena. }\end{array}$ & $\begin{array}{l}\text { Facilitar un procedimiento de demanda que propicie la obtención } \\
\text { de esta, y se evidencie la importancia del registro de los datos de } \\
\text { los productos vendidos. }\end{array}$ \\
\hline $\begin{array}{l}\text { Venta de productos similares en forma, tamaño, } \\
\text { diseño, precio y calidades en los actores de la cadena. }\end{array}$ & $\begin{array}{l}\text { Establecer programas de desarrollo de conjuntos, con la meta de } \\
\text { promover nuevos productos y tecnologías que lo faciliten. }\end{array}$ \\
\hline $\begin{array}{l}\text { Altos inventarios, ciclos, costos logísticos y } \\
\text { compras poco interrelacionados con la demanda } \\
\text { de los clientes. }\end{array}$ & $\begin{array}{l}\text { Trabajar en el proceso de planificación operativo y táctico para } \\
\text { correlacionar sus variables con la demanda de los clientes. }\end{array}$ \\
\hline $\begin{array}{l}\text { Ineficientes sistemas de gestión con los proveedores } \\
\text { de la cadena. }\end{array}$ & $\begin{array}{l}\text { Establecer programas de selección y evaluación de proveedores } \\
\text { conjuntos que permitan un mejor producto final. }\end{array}$ \\
\hline
\end{tabular}

Fuente: Autores.

\section{CONCLUSIONES}

En la actualidad, la integración de la cadena de suministros (ICS) es un factor primordial a la hora de la mejorar la competitividad de cualquier cadena y de los actores que la conforman. Es un elemento indispensable para alcázar objetivos comunes y altos niveles de posicionamiento. En esta investigación se presentó, como un caso de estudio, un análisis de integración de la cadena de suministros en la industria textil en Ecuador. El estudio incluyo el diseño de un instrumento de verificación para evaluar la integración de 96 actores, buscando proponer un conjunto de estrategias y acciones encaminadas a la mejora del sector. Además, se emplearon un conjunto de técnicas estadísticas para validar el instrumento diseñado, analizar los datos obtenidos, clusterizar las empresas del sector y medir el nivel de integración de la cadena. Los resultados obtenidos muestran que el sector textil en Ecuador, y en específico para la cadena de camisetas ecuatoriana, presenta un bajo nivel de integración. Esto constituye un reto para la mejora en las relaciones entre los socios, y la visión como cadena. Junto con la necesidad de implementación de estrategias conjuntas con el fin de obtener mayores beneficios para los actores de la cadena. La adopción de mejores prácticas que apliquen al sector textil será un factor clave que contribuirá al incremento de los niveles de ICS. Esto presenta como base la necesidad de formación del personal de los actores del objeto de estudio en las competencias básicas para el manejo de la integración. En futuras investigaciones proponemos comprar la integración de la cadena de suministro textil con diversos sectores de la industria en Ecuador y América Latina.

\section{REFERENCIAS}

[1] D. Ivanov. "Global Supply Chain and Operations Management". Springer International Publishing. DOI: 10.1007/978-3-319-242170. 2017.

[2] O.L. Wu and M.L. Chiu. "Examining supply chain collaboration with determinants 
and performance impact: Social capital, justice, and technology use perspectives". International Journal of Information Management. Vol. 39, pp. 5-19. 2018.

[3] A.d.J. Pulido-Rojano, P.A. Sánchez Sánchez and E. Melamed Varela. "Nuevas tendencias en Investigación de Operaciones y Ciencias Administrativas. Un enfoque desde estudios iberoamericanos". Colombia: Universidad Simón Bolivar. 2018.

[4] R.R. Lummus and R.J. Voturka. "Defining Supply Chain management: a historical perspective and practical guidelines". Industrial Management y Data Systems. Vol. 99 Issue 1. 1999.

[5] S.R.I. Pires and L.E. Carretero Díaz. "Gestión de la Cadena de Suministro". Primera edición en Español. ed. España. Mcgraw/Interamerica de España, S. A. U. 2007.

[6] K. Salas Navarro, H. Maiguel Mejía and J. Acevedo Chedid. "Inventory Management Methodology to determine the levels of integration and collaboration in supply chain". Ingeniare. Revista chilena de ingeniería. Vol. $25 \mathrm{~N}^{\circ}$ 2, pp. 326-337. 2017.

[7] T. Breitling. "Inter-functional coordination of purchasing and logistics: impact on supply chain performance". Supply Chain Forum: An International Journal. Vol. 20 Issue 2, pp. 71-88. 2019.

[8] J.M. Cogollo-Flórez and A.A. Correa-Espinal. "Analytical modeling of supply chain quality management coordination and integration: A literature review". Quality Management Journal. Vol. 26 Issue 2, pp. 72-83. 2019.

[9] F. Fulconis and B. Philipp. "La supply chain face aux défis de la livraison e-commerce: entre intégration et flexibilité, quelles solutions packaging?". Logistique \& Management. Vol. 27 Issue 2, pp. 132-147. 2019.

[10] S.A. Hoseini Shekarabi, A. Gharaei and M. Karimi. "Modelling and optimal lot-sizing of integrated multi-level multi-wholesaler supply chains under the shortage and limited warehouse space: generalised outer approximation". International Journal of Systems Science: Operations \& Logistics. Vol. 6 Issue 3, pp. 237-257. 2019.

[11] N. Kumar, et al. "Integrating sustainable supply chain practices with operational performance: an exploratory study of Chinese
SMEs". Production Planning \& Control. Vol. 30 Issue (5-6), pp. 464-478. 2019.

[12] Y. Yu, M. Zhang and B. Huo. "The impact of supply chain quality integration on green supply chain management and environmental performance". Total Quality Management \& Business Excellence. Vol. 30 Issue (9-10), pp. 1110-1125. 2019.

[13] M.C. Cooper, D.M. Lambert and J.D. Pagh. "Supply Chain Management: More than a New Name for Logistics". The International Journal of Logistics Management. Vol. 8 Issue (1-13). 1997.

[14] Y.P. Baez, et al. "How to achieve integration in Supply Chains? A Design Model of Integration Node in the Supply Chains". In: 5th World Production and Operations Management Conference. P\&OM Havana. 2016.

[15] Q. Zhu, H. Krikke and M.C.J. Caniëls. "Supply chain integration: value creation through managing inter-organizational learning". International Journal of Operations and Production Management. Vol. 38 Issue 1, pp. 211-229. 2018.

[16] J. Hernández Hormazabal. "Propuesta de una arquitectura para el soporte de la Planificación de la Producción Colaborativa en Cadenas de Suministro tipo árbol”. Departamento de Organización de Empresas. Universidad Politécnica de Valencia. Valencia, España, p. 752. 2011.

[17] A.W. Mackelprang, et al. "The relationship between strategic supply chain integration and performance: a meta-analytic evaluation and implications for supply chain management research". Journal of Business Logistics. Vol. 35 Issue 1, pp. 71-96. 2014.

[18] N. Sablón Cossío, et al. "Matriz de selección de estrategias de integración en las cadenas de suministro". Revista de Ingeniería Industrial. Vol. XXXVIII No 3, pp. 333-344. Septiembre-diciembre, 2017

[19] H. Bautista Santos, et al. "A fuzzy expert system for the integration of collaborative supply chains". South African Journal of Industrial Engineering. Vol. 27, pp. 234-250. 2016.

[20] F. Villareal Solís and J. Gómez Romero. "La integración en las pequeñas y medianas empresas fabricantes de muebles en la ciudad de Durango". Contaduría y Administración, p. 
227. 2009. ISSN: 0186-1042. DOI: 10.1007/ s11219-011-9141-z.

[21] N. López Lira. "Elementos de integración de microempresas comerciales en el oriente del Estado de México en los primeros años del siglo XXI". Contaduría y administración. Vol. 221, pp. 109-136. 2007.

[22] A.K. Sahu, et al. "Grey-based scorecard model for opting fruit supply bazaar locality under advanced chain of macro-micro parameter". British Food Journal. Vol. 120 Issue 1, pp. 59-79. 2018.

[23] S. Chopra and P. Meindl. "Administración de la cadena de suministro". En: Estrategia, planeación y operación. Pearson Educación ed. Vol. 1, p. 516. México. 2013.

[24] APICS. APICS dictionary 2018. American Production \& Inventory Control Society: EUA.

[25] APICS. Supply Chain Operations Reference Model. 2018.

[26] R. Alfalla Luque, C. Medina Lopez and P. Kumar Dey. "Supply chain integration framework using literature review". Production Planning \& Control. Vol. 24 Issue (8-9), pp. 800-817. 2013.

[27] J. Molka-Danielsen, P. Engelsetha and H. Wang. "Large scale integration of wireless sensor network technologies for air quality monitoring at a logistics shipping base". Journal of Industrial Information Integration. Vol. 10, pp. 20-28. 2018.

[28] W. Li and J. Chen. "Backward integration strategy in a retailer Stackelberg supply chain”. Omega (United Kingdom). Vol. 75, pp. 1339-1351. 2018.

[29] A. Fazlollahi and U. Franke. "Measuring the impact of enterprise integration on firm performance using data envelopment analysis". International Journal of Production Economics. Vol. 200, pp. 119-129. 2018.

[30] M. Yuan, E.C. Alocilja and S. Chakrabartty. "Self-Powered Wireless Affinity-Based Biosensor Based on Integration of PaperBased Microfluidics and Self-Assembled RFID Antennas". IEEE Trans Biomed Circuits Syst. Vol. 10 No 4, pp. 799-806. 2016.

[31] W. Zhou, et al. "E-Supply Chain Integration Adoption: Examination of Buyer-Supplier Relationships". Journal of Computer Information Systems. Vol. 58 Issue 1, pp. 58-65. 2018.
[32] Y. Yu, W. Xiong and Y. Cao. "A Conceptual Model of Supply Chain Risk Mitigation: The Role of Supply Chain Integration and Organizational Risk Propensity”. Journal of Coastal Research. Special Issue 73 Recent Developments of Port and Ocean Engineering, pp. 95-98. 2015. DOI: http:// dx.doi.org/10.2112/SI73-017.1.

[33] A.-H. Chiang, W.-H. Chen and S. Wu. "Does high supply chain integration enhance customer response speed?". The Service Industries Journal. Vol. 35 Issue (1-2), pp. 24-43. 2015.

[34] VICS, Linking CPFR and S\&OP: A Roadmap to Integrated Business Planning. 2010.

[35] L.J. Cronbach. "Coefficient alpha and the internal structure of tests". Psychometrika. Vol. 16 Issue 3, pp. 297-334. 1951.

[36] A. Pardo Merino and M.Á. Ruiz Diaz. "Análisis de datos con SPSS 13 Base". McGraw-Hill, Interamericana. 2006.

[37] D. George and P. Mallery. "SPSS for Windows step by step: A simple guide and reference". Fourth Edition. (11.0 update). 2003. URL: https://wps.ablongman.com/wps/media/ objects/385/394732/george4answers.pdf

[38] H. Jisu, D.E. Delorme and L.N. Reid. "Perceived Third-Person effects and consumer attitudes on prevetting and banning DTC advertising". Journal of Consumer Affairs. Vol. Vol. $40 \mathrm{~N}^{\mathrm{o}}$ 1, pp. 90-116. 2006.

[39] INEC. Metodología Directorio de Empresas y Establecimientos. 2017. URL: http://www. ecuadorencifras.gob.ec/directoriodeempresas/

[40] CAN. Decisión 702. "Sistema Andino de Estadísticas de la PYME". 2008. Lima, Perú. Secretaría General de la Comunidad Andina. URL: http://www.comunidadandina. org/Seccion.aspx

[41] P.H. Lee, et al. "A cluster analysis of patterns of objectively measured physical activity in Hong Kong". Public health nutrition. Vol. 16 Issue 8, pp. 1436-1444. 2013.

[42] IBM, IBM SPSS Statistics 21 core system user's guide: IBM Corporation Somers. New York. 2012.

[43] E. Orozco Crespo, et al. "Supply chain the T-shirt with circular economy approach. Case study in Ecuador". Proceedings of the International Conference on Industrial Engineering and Operations Management. París. 2018. 